\title{
Article
}

\section{Exploration of the effects of fin geometry and material properties on thermal performance of convective-radiative moving fins}

\author{
G. M. Sobamowo ${ }^{1}$, O. M. Kamiyo ${ }^{1}$, M. O. Salami ${ }^{1}$ and A. A. Yinusa ${ }^{1,2, *}$ \\ 1 Department of Mechanical Engineering, University of Lagos, Akoka, Lagos State, Nigeria.; \\ amikegbeminiyiprof@yahoo.com(M.G.S); bomkamiyo@unilag.edu.ng(O.M.K); mosalami@unilag.edu.ng(M.O.S); \\ ahmed-amoo.yinusa@grenoble-inp.org(A.A.Y) \\ 2 Grenoble INP Institute of Engineering, University Grenoble Alpes (Institute Polytechnique de Grenoble), France.; \\ aayinusa@unilag.edu.ng(A.A.Y) \\ * Correspondence: ahmed-amoo.yinusa@grenoble-inp.org
}

Received: 20 April 2019; Accepted: 22 August 2019; Published: 22 September 2019

\begin{abstract}
The performance of fins, commonly used as heat enhancement devices are greatly affected by both the geometry and material properties. These consideration in fin design has stimulated an extensive research interest in the recent time. In this study, investigation on the thermal responses of moving irregular porous fins with trapezoidal, concave and convex profiles of copper, aluminium, silicon nitrides and stainless steel materials is examined. The developed thermal model is solved using differential transform method (DTM). On the verification of result obtained with numerical method using Runge-Kutta, a good agreement with the solution of approximate method is achieved. In the parametric studies carried out, the effect of physical parameters such as convective-conductive, convective-radiative term, internal heat generation, porosity, surface emissivity, power index of heat transfer coefficient, Peclet number and Darcy number on the thermal behaviour of fins are examined and discussed. The comparative analysis carried out on the effect of materials on non-dimensional temperature distribution reveals that copper obtains the highest temperature while the stainless steel gets the lowest. More-so, the fins with concave geometry gives the highest volume adjusted efficiency with increase in Peclet number while that with convex profile has the least. These result output are essential and would be useful in the future design of fins with optimum size reduction and high efficiency.
\end{abstract}

Keywords: Convective-Radiative moving fin, differential transform method, fin geometry, thermal analysis.

\section{Introduction}

$\mathbf{F}$ ins are extended surfaces used for heat enhancement in a thermal system. It finds numerous application in a wide variety of industries such as electronics, automobile, aerospace, thermal plant, processing plant etc [1]. It is a known fact that the activities of some industries such as chemical, nuclear and power plant quite often involves excessive generation of large amount of heat, which requires a careful method of heat dissipation. In contrast, a close look at some other industrial applications reveals that heat enhancement into the thermal system may be required. Typical example of this is found in specialized boilers and heat exchanger equipment. Consequently, increasing demand for heat enhancement devices in the industries have provoked a lot of research interest on the different types of fin design, in order to suit ever increasing industrial need. Notably among the existing fin designs are longitudinal or straight fins, radial fins, porous fins, perforated fins, composite and laminated fins to mention but few. A survey of existing literatures reveal an extensive work on the analysis of different types of fin designs. Ghasemi et al. [2], investigated a solid and porous fin with temperature dependent heat generation using differential transform method (DTM) and found out that temperature distribution is strongly dependent on the Darcy and Rayleigh number for the porous fins and the result obtained is in good agreement with the numerical result. Kosarev [3] researched on steady state thermal analysis of rectangular fins with variable thermal conductivity using grapho-analytical method and shows the possibility of solving heat transfer problems with allowance for variation of thermal conductivity of 
the materials with temperature. Laor and Kalman [4] worked on the performance and optimum dimension of different cooling fins with temperature dependent heat transfer coefficient using theoretical numerical analysis methods and discovered that fin efficiency of spine is greater than that longitudinal fin because shape factor has less effect on fin efficiency of longitudinal fins as compared to that of spine and the optimum dimension of any fin is attained at a point where the maximum value of dissipation per mass for any fin volume and condition is attained. Taler and Taler [5], examined the steady state and transient heat transfer through fins of complex geometry using finite element finite volume method and arrived at the conclusion that the method gave a very satisfactory result, as such can be used to determine the transient response as well as steady state efficiency of fins attached to oval and circular tubes. In the work of Sun et al. [6], collocation spectral method is used to solve the non-linear heat transfer problem in a convective-radiative rectangular fins and the result obtained when verified, shows a good agreement with the analytical solutions obtained in the literature. Ganji and Dogonchi [7], carried out analytical investigation of convective heat transfer of a longitudinal fin with temperature-dependent thermal conductivity, heat transfer coefficient and heat generation using the differential transform method (DTM), the result obtained indicates that the fin tip temperature increases with an increase in heat generation gradient and also observed that the result obtained by (DTM) is in good agreement with existing numerical data. Jasim [8] examined the transient thermal response of two dimensional composite fin using the finite element technique and observed that the larger the conductivity ratio, the faster the time to reach the steady state and further demonstrated that the efficiency of the fin increase with increase in thermal conductivity ratio. Muzychka and Yovanovich [9], developed an analytical model to compute transient thermal stresses in a biomaterial system using Laplace-numerical methods and discovered that the maximum peel stress under steady state conditions shows smaller or negative stress while the transient solution shows a very large maximum stress during initial stage of the heating process of the biomaterial component. Hatami and Ganji [10] worked on the thermal analysis of a convective-radiative fin with temperature dependent thermal properties using the collocation spectral methods. The result of the study reveals an excellent agreement with the analytical result from the literature and as such, could be used as an effective technique in handling non-linear problems in science and engineering.

Based on the existing knowledge that heat conduction rate decrease from the fin base to the tip, recent fin designed shapes are varied along the direction of conduction for optimum material utilization, which further underscore the effect of fin geometry in heat enhancement process as seen in the work of Vahabzadeh et al. [11], where analytical study of porous pin fins with variable section in fully wet conditions was conducted. The result obtained shows a concave profile having the highest efficiency while the rectangular profile having the least. Hatami and Ganji [12], establishes on similar trend, on 'optimisation of longitudinal fins with different for increasing heat transfer'. Chen and Wang [13], investigated trapezoidal fins for assessment of heat sink performance and material savings. The study reveals $0.88 \%$ difference in heat transfer ratio between trapezoidal and rectangular profiles. Mosayebidorcheh et al. [14], carried out the transient analysis of longitudinal fins with internal heat generation, considering the effects of thermal conductivity and different profiles. A comparative study of longitudinal fins of rectangular, trapezoidal and concave parabolic with multiple non linearity was presented in the work of Torabi et al. [15], which further demonstrated the effect of geometry in heat enhancement. Torabi and Aziz [16], evaluates the thermal response and efficiency of convective-radiative T-shaped fins with temperature dependent thermal, heat transfer coefficient and surface emissivity and proved that T-shaped fins is significant in engineering applications. In a related work of Hatami and Ganji [17], thermal behaviour of longitudinal porous fins of different profiles of ceramic materials are analysed using the least square method, the result shows that fin of exponential shape with material composition(Si3 N4) has the highest heat transfer potentials. The effectiveness of spectral element method (SEM) is demonstrated in the study carried out by Jing et al. [18] on thermal behaviour of longitudinal porous moving fins of different profiles, a comparative analysis of the result obtained with the convex profile with the one obtained by Hatami and Ganji [17] reveals that spectral element method predicts better than the least square method. Meanwhile, there is ever increasing interest in the used of most effective method for predicting thermal responses of fins under different conditions. Hence, in this work, an attempt is made to demonstrate the effectiveness of differential transform method (DTM) in solving a thermal model with high non-linearity, by making a direct comparison with the work of Hatami and Ganji [17] and Ma et al. [18]. To the best of the Arthur's knowledge, no research work up to date has used the differential transform method (DTM) to solve heat transfer problems in a moving porous fin with irregular profiles. Hence DTM 
will be used to solve for the conductive, convective and radiative heat transfer in moving porous fins of three different geometries. The profiles under consideration are trapezoidal, concave parabolic and convex parabolic shapes. The physical model for the work will be presented and discussed. Also, parametric studies will be conducted to examine the effect of thermo-physical properties such as the Peclet number, porosity, surface emissivity coefficient, non-dimensional heat generation at ambient temperature, heat generation parameter, non-dimensional ambient temperature, power index of heat transfer coefficient, convective-conductive and radiative-conductive parameter and irregular shape on non-dimensional temperature distribution. Fin efficiency will also be examined and discussed.

\section{Physical model and the governing equations}

This study is focused on porous fins of trapezoidal (Figure 1), convex parabolic (Figure 2) and concave parabolic (Figure 3) profiles moving horizontally with constant velocity. The fins are exposed to convective and radiative environment. Therefore, the thermal analysis is based on the assumptions that the thermal processing of the porous fins is in a steady state and the porous medium is isotropic, homogenous and saturated with single phase fluid. The interaction between the fluid and the porous medium is governed by Darcy law, with the fin size thickness being smaller than in other dimensions, also the transverse Biot is very small, thus making temperature varying significantly only along the longitudinal direction. The surface of the porous fin is assumed grey and diffuse, with the solid and fluid being in local thermal equilibrium. The porous medium is assumed as dispersion medium, however, the heat transfer between internal pores and fluid is omitted in this work. The fin base is maintained at uniform temperature with no contact resistance, and the fin tip is insulated (adiabatic). Lastly, parameters such as heat transfer coefficient, surface emissivity, heat generation vary with temperature by the following equations:

$$
\begin{gathered}
h(T)=h_{b}\left(\frac{T-T_{a}}{T_{b}-T_{a}}\right)^{m}, \\
\varepsilon(T)=\varepsilon_{a}\left(1+\alpha\left(\frac{T-T_{a}}{T_{b}-T_{a}}\right)\right),
\end{gathered}
$$

and

$$
q(T)=q_{a}\left[1+c_{1}\left(\frac{T-T_{a}}{T_{b}-T_{a}}\right)+c_{2}\left(\frac{T-T_{a}}{T_{b}-T_{a}}\right)^{2}+c_{3}\left(\frac{T-T_{a}}{T_{b}-T_{a}}\right)^{3}\right] .
$$

Based on these assumptions, the energy balance on the element of porous fin is given according to energy conservation law.

Energy in the left + heat generated in the element = energy out right face + energy lost by immersed fluid as shown below

$$
\begin{aligned}
-k_{e f f} W \frac{d T}{d x}+q(T) \delta W d x= & -k_{e f f} W\left[\delta \frac{d T}{d x}+\left(\frac{d \delta}{d x} \frac{d T}{d x}+\delta \frac{d^{2} T}{d x^{2}}\right) d x\right] \\
& +2 h(T) W d x(1-\varphi)\left(T-T_{a}\right)+2 \varepsilon(T) \delta W d x(1-\varphi)\left(T^{4}-T_{a}^{4}\right) \\
& +\rho_{s} c_{p, s} \delta W d x u_{x} \frac{d T}{d x}+m c_{p f}\left(T-T_{a}\right) .
\end{aligned}
$$

Thickness of the element local fin is expressed as

$$
\delta(x)=\left(\delta_{0}-\delta_{e}\right)\left(1-\frac{x}{L}\right)^{n}+\delta_{e}
$$

Where $n=1, \frac{1}{2}, 2$ for the trapezoidal, convex parabolic and convex parabolic respectively. The mass flow rate 


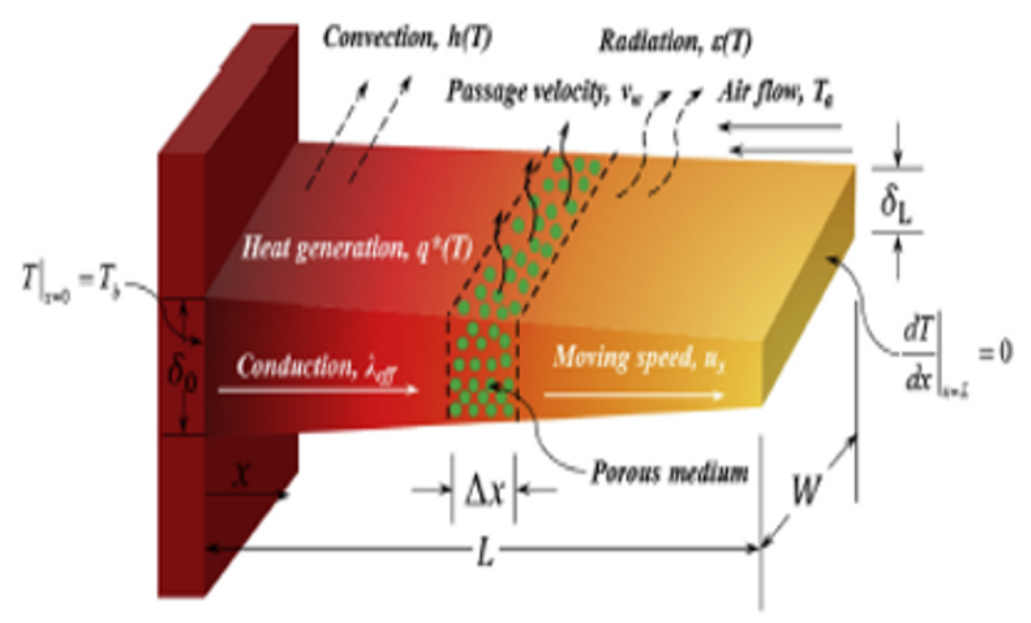

Figure 1. Trapezoidal fin profile

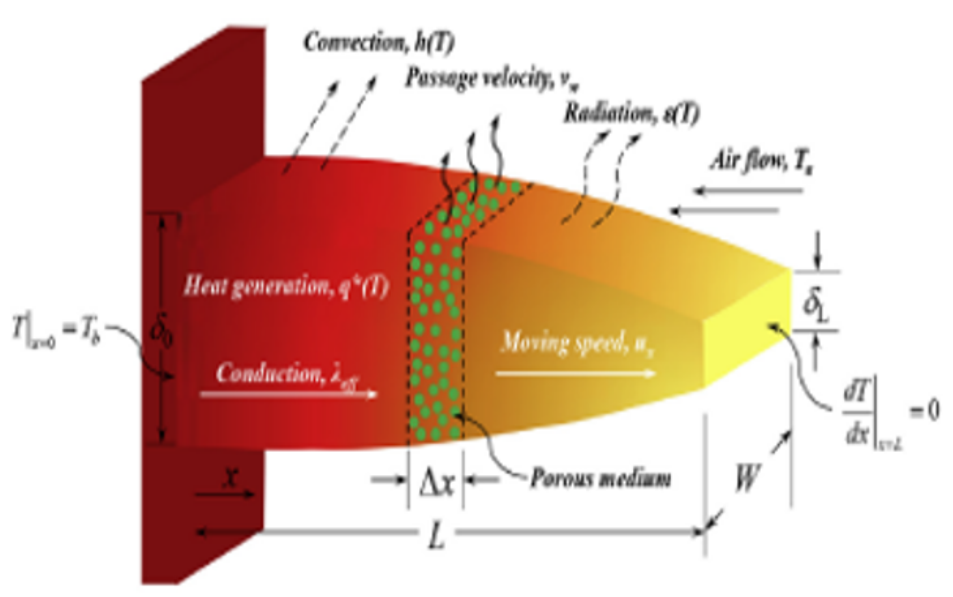

Figure 2. Convex fin profile

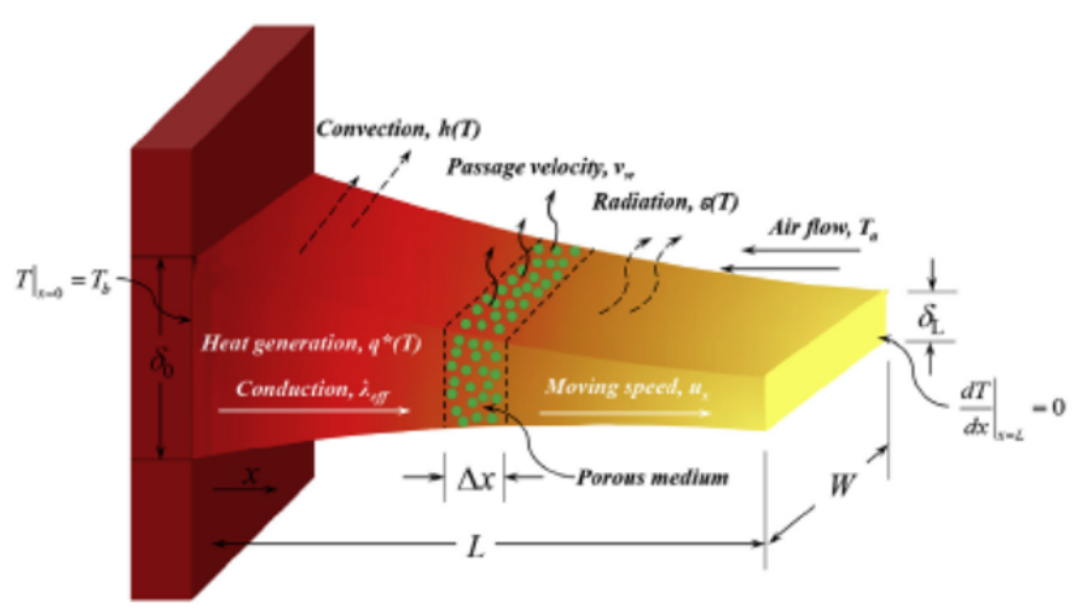

Figure 3. Concave fin profile

equation is expressed below as:

$$
m=\rho_{f} v_{w} W d x,
$$


where $v_{w}$ is the fluid velocity

$$
v_{w}=\frac{k g \beta}{v_{f}}\left(T-T_{a}\right)
$$

where $k=$ permeability of porous fin, $g=$ gravitational acceleration, $\beta=$ coefficient of thermal expansion and $v_{f}=$ kinematic viscosity of the fluid.

The resulting equation below is obtained when Equations (1), (2), (3), (5), (6) and (7) are substituted in Equation (4)

$$
\begin{aligned}
& k_{e f f} \delta \frac{d^{2} T}{d x^{2}}+k_{e f f} \frac{d \delta}{d x} \frac{d T}{d x}+\delta q_{a}\left[1+c_{1}\left(\frac{T-T_{a}}{T_{b}-T_{a}}\right)+c_{2}\left(\frac{T-T_{a}}{T_{b}-T_{a}}\right)^{2}+c_{3}\left(\frac{T-T_{a}}{T_{b}-T_{a}}\right)^{3}\right] \\
& =2(1-\varphi) h_{b}\left(\frac{T-T_{a}}{T_{b}-T_{a}}\right)^{m}\left(T-T_{a}\right)+2(1-\varphi) \varepsilon_{b}(1+\alpha)\left(\frac{T-T_{a}}{T_{b}-T_{a}}\right) \sigma\left(T^{4}-T_{a}^{4}\right)+\delta \rho_{s} c_{p, s} u_{x} \frac{d T}{d x} \\
& +\rho_{f} c_{p, f} \frac{k g \beta}{v_{f}}\left(T-T_{a}\right)^{2}
\end{aligned}
$$

where, this relationship holds for effective thermal conductivity, fluid thermal conductivity and solid conductivity.

$$
k_{e f f}=\varphi k_{f}+(1-\varphi) k_{s} .
$$

Similarly as referenced in [13], the following non dimensional parameters are adopted,

$$
\begin{array}{r}
X=\frac{x}{L}, \psi=\frac{\delta_{0}}{L}, \theta=\frac{T}{T_{b}}, \quad k_{r}=\frac{k_{e f f}}{k_{f}} R a=G r P r, P r=\frac{\rho_{f} c_{p, f} v_{f}}{k_{f}}, N_{c c}=\frac{2 h_{b} L^{2}}{k_{e f f} \delta_{0}}, D a=\frac{K}{\delta_{0}^{2}} \\
N_{r c}=\frac{2 \varepsilon_{a} \sigma T_{b}^{3} L^{3}}{k_{e f f} \delta_{0}}, P e=\frac{\rho_{s} c_{p, s} u_{x} L}{k_{e f f}}, \quad G r=\frac{\beta g T_{b} \delta_{0}^{3}}{v_{f}^{2}}, \quad s_{h}=\frac{D a R a}{\psi^{2} k_{r}}, Q_{a}=\frac{L^{2} q_{a}}{T_{b} k_{e f f}} .
\end{array}
$$

Substituting the non-dimensional quantities in Equation (8), the resulting non-dimensional energy equation is obtained.

$$
\begin{aligned}
\frac{\delta}{\delta_{0}} \frac{d^{2} \theta}{d x^{2}}+\frac{d}{d x}\left(\frac{\delta}{\delta_{0}}\right) \frac{\theta}{d x}= & {\left[N_{c c}(1-\varphi) 1\left[\frac{\theta-\theta_{a}}{1-\theta_{a}}\right]^{m}\left(\theta-\theta_{a}\right)+N_{r c}(1-\varphi)\left[1+\alpha\left[\frac{\theta-\theta_{a}}{1-\theta_{a}}\right]\right]\left(\theta^{4}-\theta_{a}^{4}\right)\right.} \\
& \left.+\frac{\delta}{\delta_{0}} P e \frac{d \theta}{d x}+S_{h}\left(\theta-\theta_{a}\right)^{2}-Q_{a} \frac{\delta}{\delta_{0}}\left[1+c_{1}\left(\frac{\theta-\theta_{a}}{1-\theta_{a}}\right)+c_{2}\left(\frac{\theta-\theta_{a}}{1-\theta_{a}}\right)^{2}+c_{3}\left(\frac{\theta-\theta_{a}}{1-\theta_{a}}\right)^{3}\right]\right] .
\end{aligned}
$$

The boundary condition for Equation (11) is shown below:

$$
\begin{gathered}
\left.\theta\right|_{x=0}=1 . \\
\left.\frac{d \theta}{d X}\right|_{x=1}=0 .
\end{gathered}
$$

\section{Concept and Application of differential transformation method}

The differential transform method (DTM) was first used by Zhou [14]. The concept described $f(x)$ to be an analytical function in the domain $D$, while $x=x_{j}$ represent any point in the domain. The Taylor's series expansion of $f(x)$ which has its center point as $x_{j}$ can be expressed as follows:

$$
f(x)=\sum_{k=0}^{\infty} \frac{\left(x-x_{j}^{k}\right)}{k !}\left[\frac{d^{k} f(x)}{d x^{k}}\right]_{x=x_{j}}, \quad \forall \in D .
$$


A case where $x_{j}=0$, Equation (14) reduces to McLaurin series expansion of $f(x)$ which is expressed as

$$
f(x)=\sum_{k=0}^{\infty} \frac{x^{k}}{k !}\left[\frac{d^{k} f(x)}{d x^{k}}\right]_{x=0}, \quad \forall \in D
$$

In the work of Zhou [14], the differential transformation of the function $f(x)$ is expressed as:

$$
F(k)=\sum_{k=0}^{\infty} \frac{Y^{k}}{k !}\left[\frac{d^{k} f(x)}{d x^{k}}\right]_{x=0} .
$$

where $f(x)$ represents the original analytical function and $F(k)$ is the transformed function. The differential spectrum of $F(k)$ is confined within an interval $x \in[0, Y]$, where $Y$ is a constant value. Therefore, the differential inverse transform of $F(k)$ is given as

$$
f(x)=\sum_{k=0}^{\infty}\left(\frac{x}{Y}\right)^{k} F(k) .
$$

The more the number of terms of the series expansion generated, the higher the accuracy of result. Hence, we approximate $f(x)$ by a finite series, Equation (17) becomes

$$
f(x)=\sum_{k=0}^{n}\left(\frac{x}{Y}\right)^{k} F(k),
$$

where $\infty=n$ in the case of a finite series. More so, the values of function $F(k)$ and the values of argument $k$ are referred to as discrete. Some of the useful mathematical function and their differential transformation are given in the Table 1 [1].

Table 1. Transformations of some functions

\begin{tabular}{||lll||}
\hline 1. & Original function & Transformed function \\
2. & $f(x)=u(x) \pm v(x)$ & $F(x)=U(x) \pm V(x)$ \\
3. & $f(x)=\frac{d y(x)}{d x}$ & $F(x)=\alpha U(x)$ \\
4. & $f(x)=\frac{d^{2} y(x)}{s x^{2}}$ & $F(x)=(k+1) F(k+1)$ \\
5. & $f(x)=\frac{d^{n} y(x)}{d x^{n}}$ & $F(x)=(k+1)(k+2) \ldots(k+n) F(k+n)$ \\
6. & $f(x)=u(x) v(x)$ & $F(k)=\sum$ lu $)$ \\
7. & $f(x)=a(\operatorname{constant})$ & $F(k)=\alpha \delta(k)$ where $\delta(k)=\left\{\begin{array}{l}\text { lif } k=0 \\
\text { if } k \neq 0\end{array}\right.$ \\
8. & $f(x)=x$ & $F(k)=\delta(k-1)$ \\
9. & $f(x)=x^{n}$ & $F(k)=\delta(k-n)$ \\
10. & $f(x)=\exp (\lambda x)$ & $F(k)=\frac{\lambda^{k}}{k !}$ \\
11. & $f(x)=(1+x)^{n}$ & $F(k)=\frac{n(n-1) \ldots(n-k+1)}{k !}$ \\
12. & $f(x)=\sin (\omega x+\alpha)$ & $F(k)=\frac{\omega^{k}}{k !} \sin \left(\frac{\pi K}{2 !}+\alpha\right)$ \\
13. & $f(x)=\cos (\omega x+\alpha)$ & $F(k)=\frac{\omega^{k}}{k !} \cos \left(\frac{\pi K}{2 !}+\alpha\right)$ \\
\hline
\end{tabular}

Applying the DTM recursive relation to the governing equation, we have

$$
\delta_{t ; k}=\delta_{0} \sigma(k)-\frac{\sigma(k-1) \delta_{0}}{L}+\frac{\sigma(k-1) \delta_{e}}{L},
$$




$$
\begin{aligned}
& \sum_{l=0}^{k} \Theta_{l}(k-l+1) F_{k-l+1}-\theta_{a}(k+1) F_{k+1}-m \sum_{l=0}^{k} F_{l}(k-l+1) \Theta_{k-l+1}=0, \\
& 0=\frac{\sum_{l=0}^{k} \delta_{t ; k}(k-l+1)(k-l+2) \Theta_{k-l+2}}{\delta_{0}}-\frac{\sum_{l=0}^{k}(l+1) \delta_{t ; l+1}(k-l+1) \Theta_{k-l+1}}{\delta_{0}} \\
& -N_{c c}(1-\varphi)\left(\sum_{l=0}^{k} F_{l} \Theta_{k-l}-\theta_{a} F_{k}\right)-N_{r c}(1-\varphi)\left[\sum_{r=0}^{k}\left(\sum_{p=0}^{r}\left(\sum_{l=0}^{p} \Theta_{l} \Theta_{p-l} \Theta_{r-p} \Theta_{k-r}\right)\right)-\theta_{a}^{4} \sigma(k)\right. \\
& +\frac{\alpha\left(\sum_{s=0}^{k}\left(\sum_{r=0}^{s}\left(\sum_{p=0}^{r}\left(\sum_{l=0}^{p} \Theta_{l} \Theta_{p-l} \Theta_{r-p} \Theta_{s-r} \Theta_{k-s}\right)\right)\right)\right)}{1-\theta_{a}} \\
& -\alpha\left(\frac{\theta_{a} \sum_{r=0}^{k}\left(\sum_{p=0}^{r}\left(\sum_{l=0}^{p} \Theta_{l} \Theta_{p-l} \Theta_{r-p} \Theta_{k-r}\right)\right)}{1-\theta_{a}}\right) \\
& \left.-\alpha\left(\frac{\theta_{a}^{4} \Theta_{k}+\theta_{a}^{5} \sigma(k)}{1-\theta_{a}}\right)\right]-\frac{P_{e} \sum_{l=0}^{k} \delta_{t ; k}(k-l+1) \Theta_{k-l+1}}{\delta_{0}}-S_{h}\left(\sum_{l=0}^{k} \Theta_{l} \Theta_{k-l}-2 \theta_{a} \Theta_{k}+\theta_{a}^{2} \sigma(k)\right) \\
& +\frac{Q_{a}}{\delta_{0}}\left[\delta_{t ; k}+\frac{c_{1}\left(\sum_{l=0}^{k} \delta_{t ; k} \Theta_{k-l}-\delta_{t ; k} \theta_{a}\right)}{1-\theta_{a}}+\frac{c_{2}\left(\sum_{l=0}^{k}\left(\sum_{r=0}^{l} \delta_{t ; k} \Theta_{l-r} \Theta_{k-l}\right)-2 \theta_{a} \sum_{l=0}^{k} \delta_{t ; k} \Theta_{k-l}+\delta_{t ; k} \theta_{a}^{2}\right)}{\left(1-\theta_{a}\right)^{2}}\right. \\
& +\frac{c_{3}\left(\sum_{r=0}^{k}\left(\sum_{p=0}^{r}\left(\sum_{l=0}^{p} \delta_{t ; k} \Theta_{p-l} \Theta_{r-p} \Theta_{k-r}\right)\right)-3 \theta_{a} \sum_{l=0}^{k}\left(\sum_{r=0}^{l} \delta_{t ; k} \Theta_{l-r} \Theta_{k-l}\right)\right)}{\left(1-\theta_{a}\right)^{3}} \\
& \left.+\frac{c_{3}\left(3 \theta_{a}^{2} \sum_{l=0}^{k} \delta_{t ; k} \Theta_{k-l}-\delta_{t ; k} \theta_{a}^{3}\right)}{\left(1-\theta_{a}\right)^{3}}\right]
\end{aligned}
$$

Solving Equations (19), (20), (21) with the transformed boundary conditions, the required series solution is represented as;

$$
\theta(X)=\sum_{\xi=0}^{N} \Theta_{\xi} X^{\xi}
$$

\section{Verification}

Table 2 depicted the comparative result obtained from silicon nitride of convex cross-section using the four different methods. The agreement of DTM employed in the present study with numerical as well as already published works [18], verifies the scheme.

\subsection{The model efficiency determination}

The following expression is used to define the efficiency (i.e., the ratio of actual heat transfer rate to the ideal heat transfer if entire fin area is maintained at the base temperature)

$$
\eta=\frac{\int_{0}^{1}\left(N_{c c}(1-\varphi) \frac{\left(\theta-\theta_{a}\right)^{m+1}}{\left(1-\theta_{a}\right)^{m}}+N_{r c}(1-\varphi)\left(1+\frac{\alpha\left(\theta-\theta_{a}\right)}{1-\theta_{a}}\left(\theta^{4}-\theta_{a}^{4}\right)\right)\right) d x+\int_{0}^{1}\left(\frac{\delta}{\delta_{0}} P_{e}\left(\theta-\theta_{a}\right)+S_{h}\left(\theta-\theta_{a}\right)^{2}\right) d x}{N_{c c}(1-\varphi)\left(1-\theta_{a}\right)+N_{r c}(1-\varphi)(1+\alpha)\left(1-\theta_{a}^{4}\right)+P_{e}\left(1-\theta_{a}\right) \int_{0}^{1} \frac{\delta}{\delta_{0}} d x+S_{h}\left(1-\theta_{a}\right)^{2}} .
$$


Table 2. Verification of DTM

\begin{tabular}{||c|ccc|c||}
\hline $\mathrm{X}$ & \multicolumn{3}{|c||}{$\theta$} & \\
\hline & LSM & SEN & DTM & Numerical Solution \\
\hline \hline 1 & 0.885112812 & 0.885112628 & 0.848340571 & 0.84965339 \\
0.9 & 0.886749804 & 0.886749648 & 0.8505218 & 0.852305597 \\
0.8 & 0.891018373 & 0.891018069 & 0.85666425 & 0.85927805 \\
0.7 & 0.897194237 & 0.897193917 & 0.866233388 & 0.869518003 \\
0.6 & 0.904872207 & 0.904871474 & 0.878778309 & 0.882358962 \\
0.5 & 0.913966193 & 0.913965953 & 0.893924218 & 0.897381433 \\
0.4 & 0.924709201 & 0.924708212 & 0.911365183 & 0.914332606 \\
0.3 & 0.937653337 & 0.937653293 & 0.93085726 & 0.933078485 \\
0.2 & 0.9536698 & 0.953669404 & 0.952212099 & 0.953575046 \\
0.1 & 0.97394889 & 0.973948376 & 0.975291078 & 0.975851246 \\
0.0 & 1.0000000000 & 1.0000000000 & 1.0000000000 & 1.0000000000 \\
\hline
\end{tabular}

\section{Analysis of result and discussion}

Based on the numerical research performed by Jing Ma et al. [18], the values of these physical parameters are adopted

$$
\begin{gathered}
\psi=0.1, \varphi=0.8, \alpha=0.6, \quad m=2, P_{e}=1.0, \quad N_{c c}=1.0 \\
N_{r c}=1.0, \quad D a=10^{-5}, \theta_{a}=0.3, \quad Q_{a}=0.1, \quad c_{1}=c_{2}=c_{3}=0.2 .
\end{gathered}
$$

Thermal physical properties of the selected materials used are the same as in [18]. Figure 4 depicts the effect of

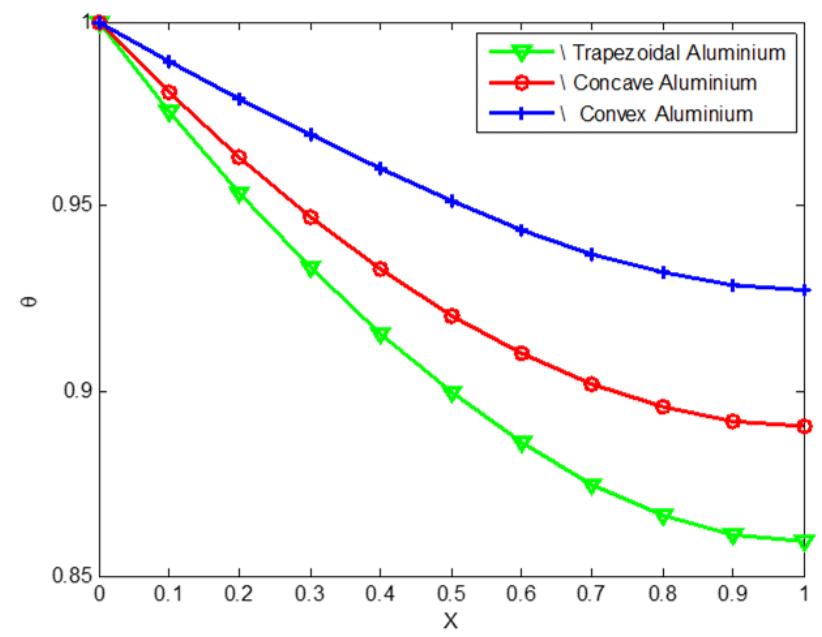

Figure 4. Effect of geometries of Aluminium porous fins on non-dimensional temperature distribution

different geometry on non-temperature distribution of Aluminium porous fins, with convex parabolic having the highest temperature distribution, while the trapezoidal having the least, this may be due to their respective convective and radiative surface areas, such that the more the surface area available for heat dissipation the lower the lower the non-dimensional heat distribution in the porous fin. This shows that the trapezoidal fins has the widest surface area while the convex parabolic has the least. In Figures 5, 6 and 7, the effect of temperature distribution on different materials of fin with trapezoidal, concave and convex geometry is shown.

It is observed that in all three geometries considered, the trend is the same, that is, copper porous fin has the highest temperature while stainless steel has the least. The is due to higher thermal conductivity of copper material as compared to that of Aluminium, silicon nitrides and stainless steel material used. The lowest 


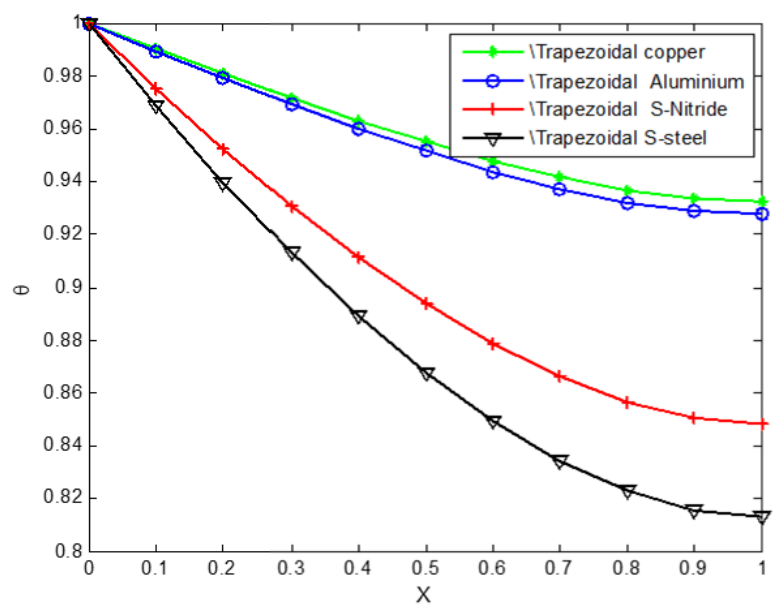

Figure 5. Effect of different materials on non-dimensional temperature distribution of fin with Trapezoidal profile

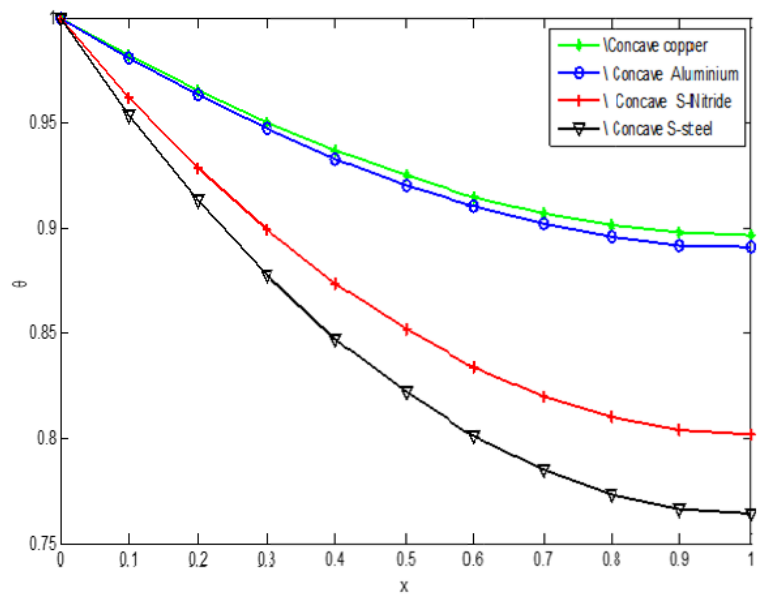

Figure 6. Effect of different materials on non-dimensional temperature distribution of fin with concave profile

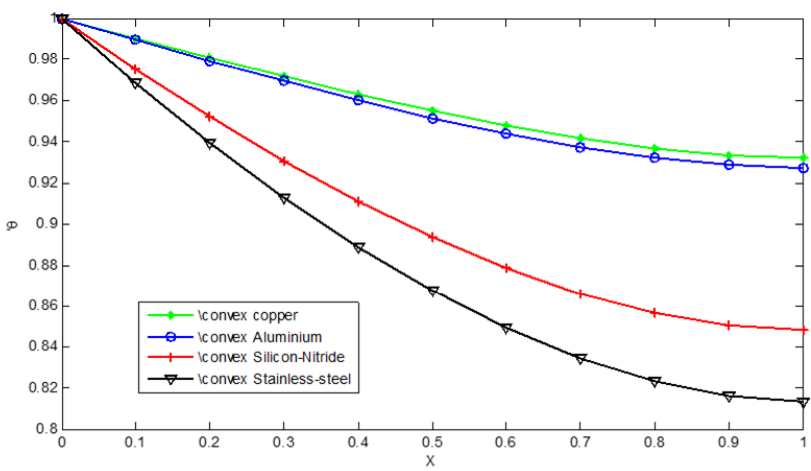

Figure 7. Effect of different materials on non-dimensional temperature distribution of fin with convex profile

temperature recorded for the stainless steel is on account of its lowest thermal conductivity and highest thermal resistance. The effect of different values of surface emissivity constant on the temperature distribution of trapezoidal silicon nitride porous fin is depicted in Figure 8. Since surface emissivity show a direct relationship between the rates of variation in the surface emissivity with temperature. As it increases, radiative heat dissipation increases from the porous surface of the fin and the non-dimensional temperature distribution increases accordingly. The effect of different power index of heat coefficient in a concave aluminium porous fin is displayed in Figure 9. The trend shows that the higher the value of power index of heat coefficient, the higher 


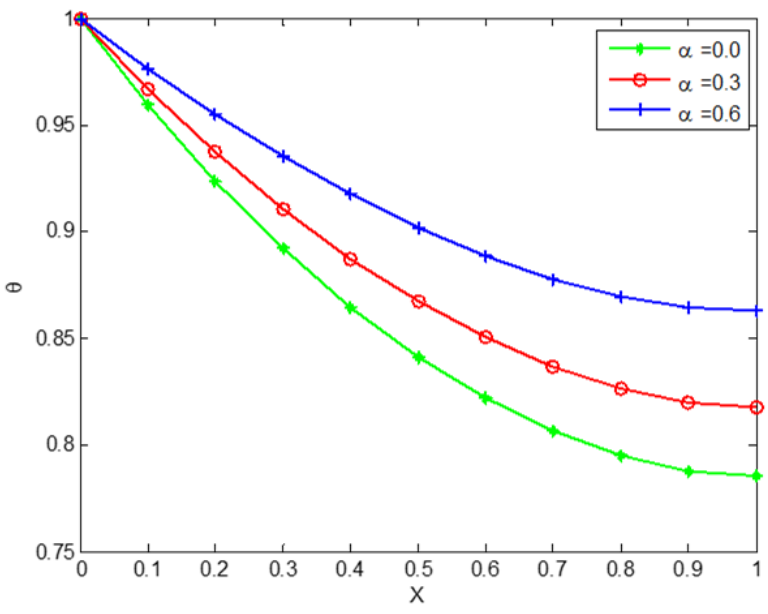

Figure 8. Effect of different value of surface emissivity on temperature distribution of trapezoidal silicon nitride porous fin

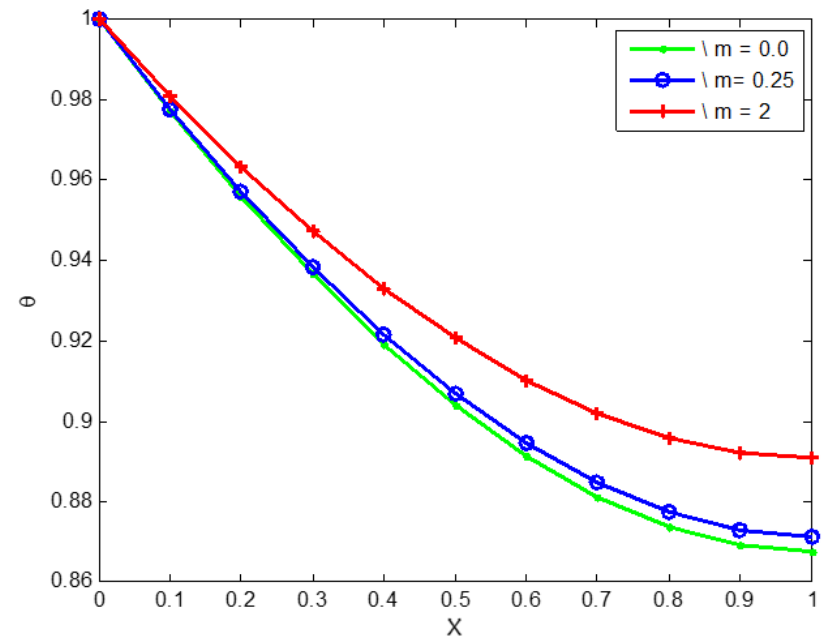

Figure 9. Effect of different values of power index of heat coefficient on temperature distribution of Concave Aluminium porous fin

the non-dimensional heat distribution in the Aluminium porous fin. The curve marked $m=0$ represents temperature distribution with forced convection model, while the curve marked with $m=0.25$ represent temp distribution with laminar natural convection model and the curve marked with $m=2$ represent non dimensional temperature distribution with nucleated boiling model which lead to heat loss reduction. Figure 10 shows a rapid increase in temperature distribution with increase in peclet number for the convex stainless steel porous fins. The trend shows that as the peclet number increases, the non-dimensional temperature becomes higher. This is due to the fact that the porous fin move faster as the peclet number increases and the heat transfer time between the fin surface and the ambient fluid get shorter, while leads to rise in temperature distribution. This phenomenon is established in the work of Sivaraj et al. [19], where the increase in Peclet number is associated with decrease in shear stress. In a similar study, carried out by Makinde and Animashaun [20], it was established that Peclet number is a decreasing property of diffusion gradient of mobile microorganism along the wall. The effect of internal heat generation Qa and the effect of heat generation parameter (c1, c2 and c3) on the convex copper porous fin is examined in Figure 11 and Figure 12 respectively. The trend shows that Increase in internal heat generation $(\mathrm{Qa})$ and heat generation parameter lead to increase in non-dimensional temp distribution. This is due to the fact that the more the heat generation the higher the non-dimension temp distribution, because fin must dissipate larger heat to the ambient environment. Non-dimensional temperature increase with increase in porosity as depicted in Figure 13. As porosity increases the heat transfer rate decreases and the effective thermal conductivity of the porous fin decreases as well due 


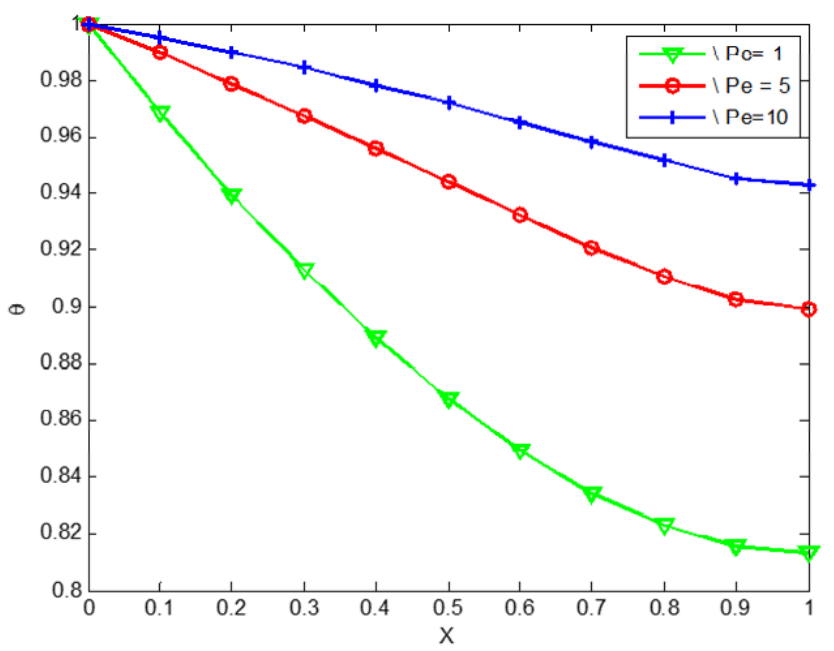

Figure 10. The effect of Peclet on the temperature distribution of a convex copper porous fins

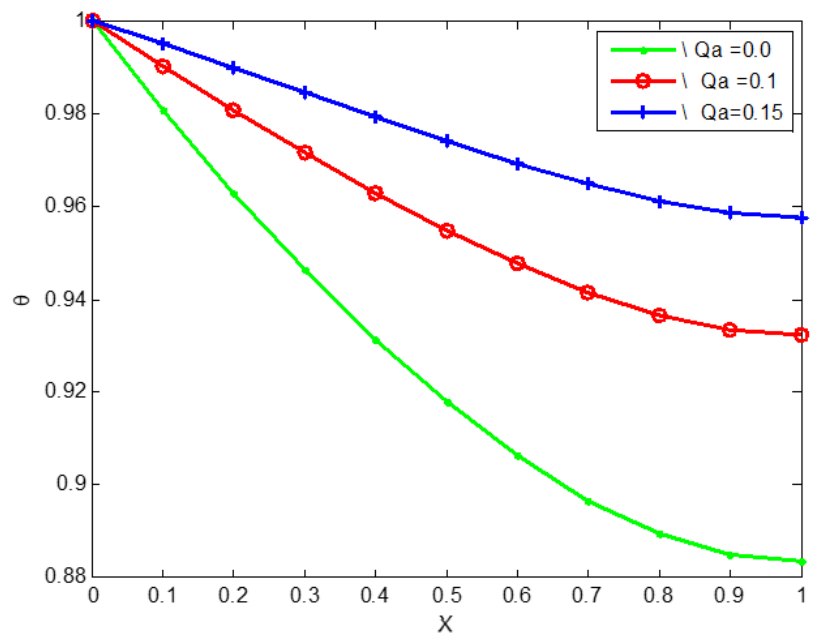

Figure 11. The effect of internal heat generation on the temperature distribution of a convex copper porous fins

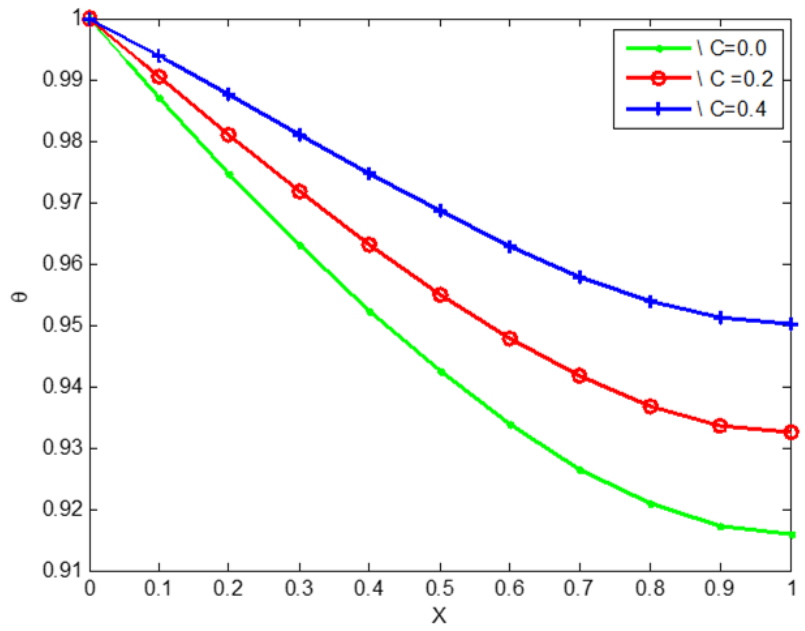

Figure 12. The effect of heat generation parameter on the temperature distribution of a convex copper porous fins

to void increment. However, the more the porosity the more the convective and radiative heat enhancement capacity of the fin due to large surface area. In Figure 14, the effect of convective conductive parameter (Ncc) 


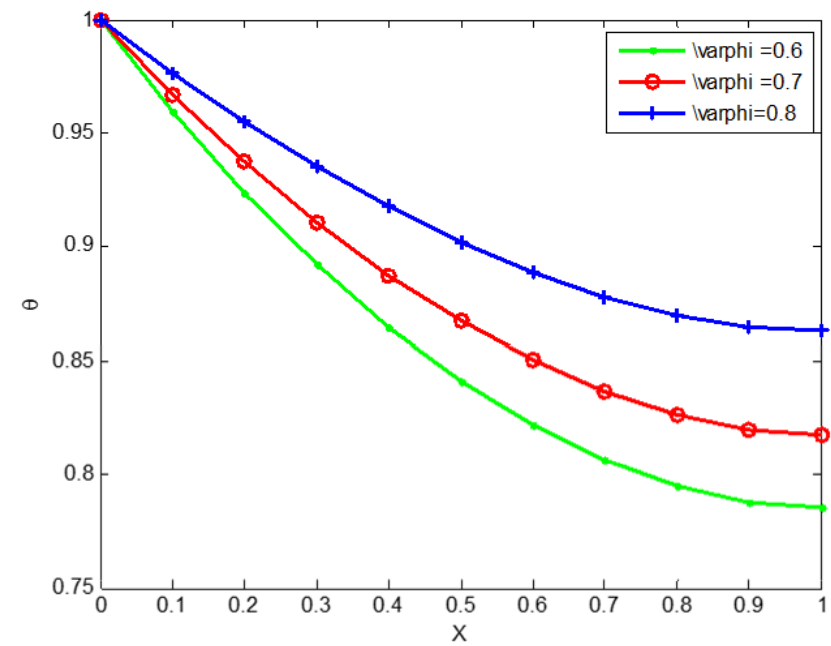

Figure 13. The effect of porosity on the non-dimensional temperature distribution for trapezoidal copper fin

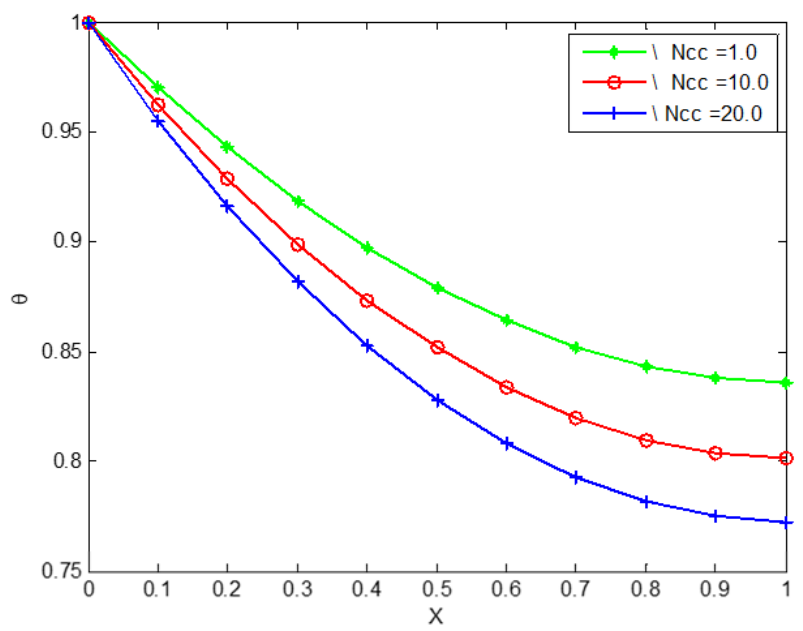

Figure 14. The effect of convective conductive parameter (Ncc) on the temperature distribution of a silicon nitride porous fin

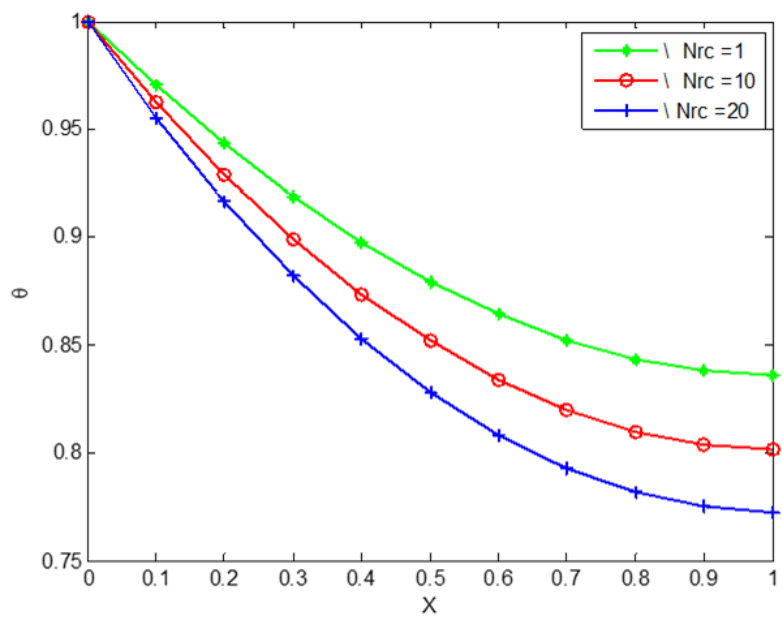

Figure 15. The effect of convective radiative parameter $(\mathrm{Nrc})$ on the temperature distribution of a silicon nitride porous fin

and in Figure 15, radiative conductive parameter Nrc is examined. Convective conductive parameter (Ncc) connotes ratio of convective heat dissipation to that of conductive heat transfer. As convective conductive 
parameter (Ncc) decrease from 10-1, convective heat loss from the surface of the porous fin decreases. In contrast, As radiative-conductive parameter Nrc decreases, the non- dimensional temperature increases, this is due to the fact that radiative heat loss is directly proportional to the difference in the fourth power of fin temperature and ambient temperature.

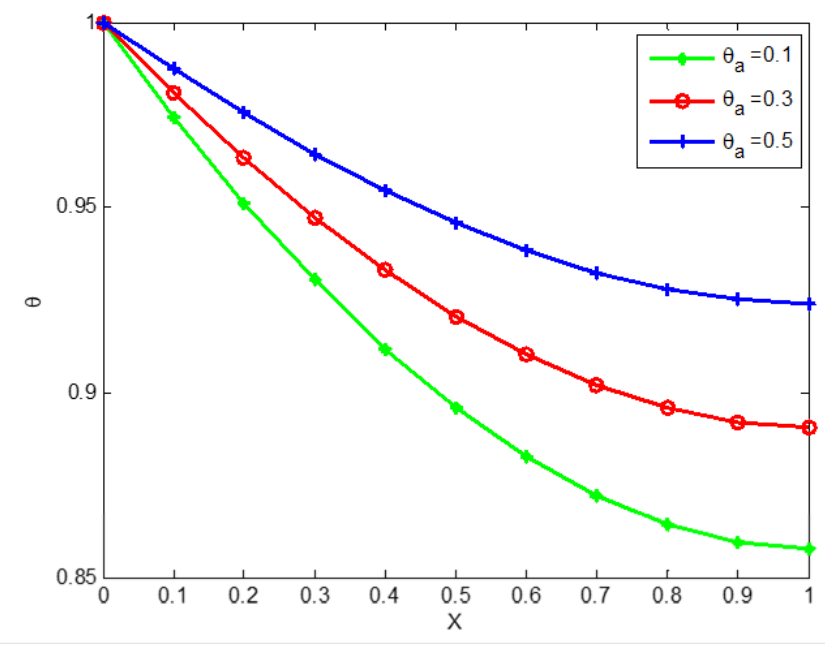

Figure 16. Non-dimensional Temperature in concave Aluminium porous fin different ambient temperature

The trend in Figure 16 shows that, as ambient temperature increases, the non-dimensional Temperature distribution in concave Aluminium porous fin increases as well due to the reduction in the temperature differentials between the fins and the environment. Figure 17 shows the trend of variation in efficiency

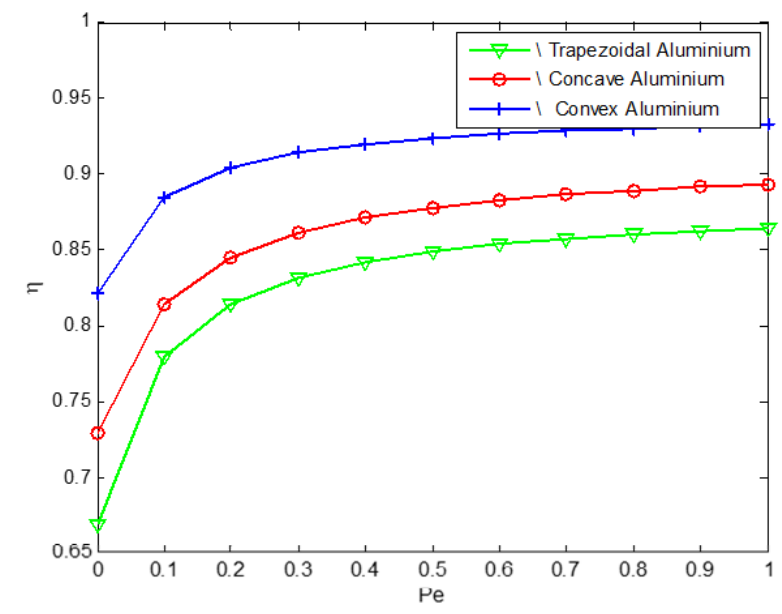

Figure 17. Variation in efficiency with Peclect number for Aluminium porous fin

with Peclect number for Aluminium porous fins. The trend shows an increase in efficiency with increase in Peclet number, with the convex Aluminium has the highest efficiency, followed by the concave profile and the least efficiency is recorded for the trapezoidal profile. However, this result does not take into consideration the difference in material consumption of different irregular profile, as such, may not provide good basis for comparison. For the same thickness and length concave parabolic porous fin consume less materials than the concave parabolic porous fin and the trapezoidal parabolic porous fin. Hence a volume adjusted efficiency is used instead. Figure 18 depicts the Variation in volume adjusted efficiency with peclect number for Aluminium porous fins. The trend shows an increase in volume adjusted efficiency with peclet number, with concave parabolic porous fin having the highest volume adjusted efficiency, followed by the convex parabolic porous fin and the least volume adjusted efficiency ids recorded in trapezoidal porous fin In Figure 19 copper porous fins displayed the same trend in volume adjusted efficiency as compared with Aluminium porous fin in Figure 18. In Figure 20, stainless steel porous fins displayed the same trend in volume adjusted efficiency as 


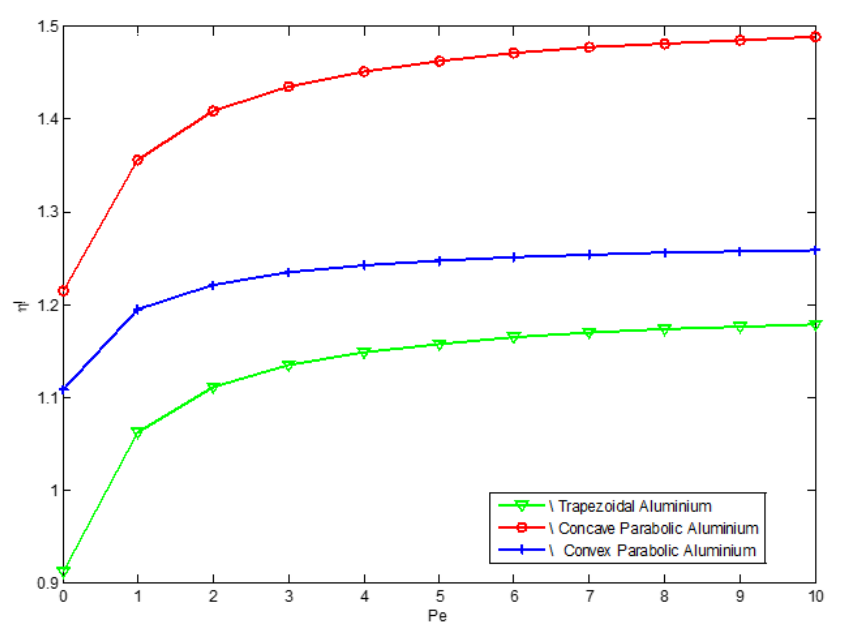

Figure 18. Variation in volume adjusted efficiency with peclect number for Aluminium porous fins

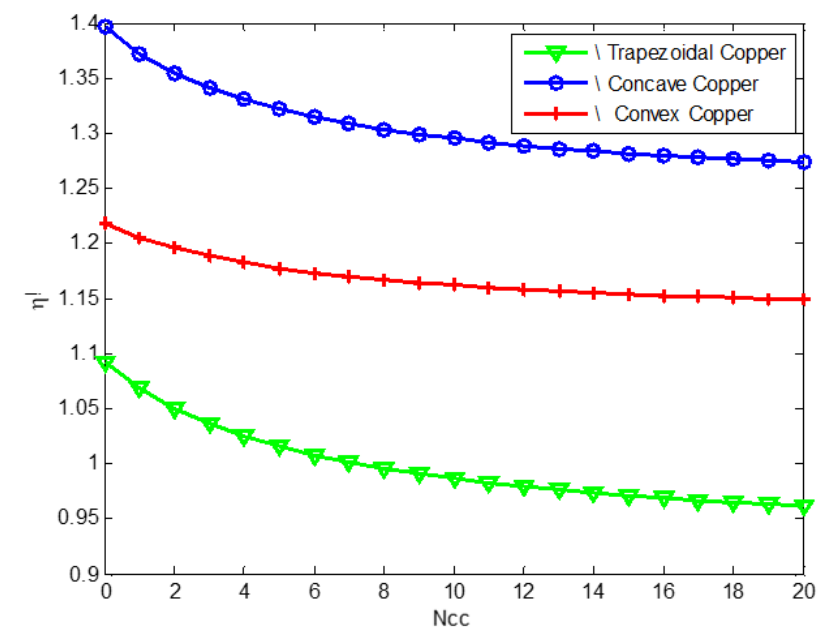

Figure 19. Variation in volume adjusted efficiency with peclect number for copper porous fins

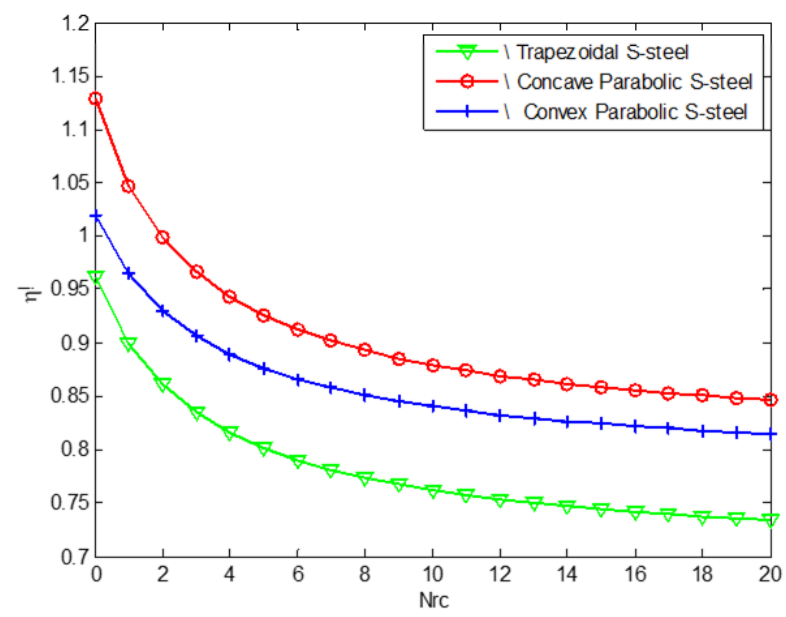

Figure 20. Variation in volume adjusted efficiency with peclect number for stainless steel porous fins

compared with both Aluminium porous fin in Figure 18. Copper porous fin in Figure 19. Thus, it can be safely concluded that volume adjusted efficiency which takes into consideration equal volume of different geometry in determining the efficiency of their heat enhancement capacity is a good basis for comparing the efficiency of fins of different geometry. 


\section{Conclusion}

The comparative analysis carried out on the effect of materials on non-dimensional temperature distribution revealed that copper fins has the highest temperature while the stainless steel gets the lowest. More so, the fins with concave geometry gives the highest volume adjusted efficiency with increase in Peclet number while that with convex profile has the least. It is envisage that the obtained results would be useful in the future design of fins with optimum size reduction and high efficiency.

Author Contributions: All authors contributed equally to the writing of this paper. All authors read and approved the final manuscript.

Conflicts of Interest: "The authors declare no conflict of interest."

\section{References}

[1] Taler, D., \& Taler, J. (2014). Steady-state and transient heat transfer through fins of complex geometry. Archives of thermodynamics, 35(2), 117-133.

[2] Ghasemi, S. E., Valipour, P., Hatami, M., \& Ganji, D. D. (2014). Heat transfer study on solid and porous convective fins with temperature-dependent heat generation using efficient analytical method. Journal of Central South University, 21(12), 4592-4598.

[3] Kosarev, D. A. (1965). Steady-state heat transfer through a wall with longitudinal rectangular fins and variable thermal conductivity. Journal of Engineering Physics and Thermophysics, 9(6), 482-484.

[4] Laor, K., \& Kalman, H. (1996). Performance and optimum dimensions of different cooling fins with a temperature-dependent heat transfer coefficient. International journal of heat and mass transfer, 39(9), 1993-2003.

[5] Taler, D., \& Taler, J. (2014). Steady-state and transient heat transfer through fins of complex geometry. Archives of thermodynamics, 35(2), 117-133.

[6] Sun, Y., Ma, J., Li, B. W., \& Guo, Z. (2015). "Thermal analysis of a convective-radiative fin with temperature dependent properties by the collocation spectral method.," in Proceedings of CHT-15 ICHMT International Symposium on Advances in Computational Heat Transfer, New Brunswick, NJ, USA.

[7] Ganji, D. D., \& Dogonchi, A. S. (2014). Analytical investigation of convective heat transfer of a longitudinal fin with temperature-dependent thermal conductivity, heat transfer coefficient and heat generation. International Journal of Physical Sciences, 9(21), 466-474.

[8] Jasim, A. M. A. (2008). Heat transfer from composite material extended surfaces heat sink. Iraqi journal of mechanical and material engineering, 8(1), 43-58.

[9] Muzychka, Y. S., \& Yovanovich, M. M. (1996). Analytic models to compute transient thermal stresses in bimaterial systems. in International electronics packaging conference., Austin, Texas, USA.

[10] Hatami, M., \& Ganji, D. D. (2014). Thermal behavior of longitudinal convective-radiative porous fins with different section shapes and ceramic materials (SiC and Si3N4). Ceramics International, 40(5), 6765-6775.

[11] Vahabzadeh, A., Ganji, D. D., \& Abbasi, M. (2015). Analytical investigation of porous pin fins with variable section in fully-wet conditions. Case Studies in Thermal Engineering, 5, 1-12.

[12] Ganji, D. \& Hatami, H. (November 2015). "Optimization of longitudinal fins with different geometries for increasing the heat transfer," in Proceedings of ISER 10th International Conference, Kuala Lumpur, Malaysia.

[13] Chen, H. L., \& Wang, C. C. (2016). Analytical analysis and experimental verification of trapezoidal fin for assessment of heat sink performance and material saving. Applied Thermal Engineering, 98, 203-212.

[14] Mosayebidorcheh, S., Farzinpoor, M., \& Ganji, D. D. (2014). Transient thermal analysis of longitudinal fins with internal heat generation considering temperature-dependent properties and different fin profiles. Energy conversion and management, 86, 365-370.

[15] Torabi, M., Aziz, A., \& Zhang, K. (2013). A comparative study of longitudinal fins of rectangular, trapezoidal and concave parabolic profiles with multiple nonlinearities. Energy, 51, 243-256.

[16] Torabi, M., \& Aziz, A. (2012). Thermal performance and efficiency of convective-radiative T-shaped fins with temperature dependent thermal conductivity, heat transfer coefficient and surface emissivity. International Communications in Heat and Mass Transfer, 39(8), 1018-1029.

[17] Hatami, M., \& Ganji, D. D. (2014). Thermal behavior of longitudinal convective-radiative porous fins with different section shapes and ceramic materials (SiC and Si3N4). Ceramics International, 40(5), 6765-6775.

[18] Ma, J., Sun, Y., \& Li, B. (2017). Simulation of combined conductive, convective and radiative heat transfer in moving irregular porous fins by spectral element method. International Journal of Thermal Sciences, 118, 475-487. 
[19] Sivaraj, R., Animasaun, I. L., Olabiyi, A. S., Saleem, S., \& Sandeep, N. (2018). Gyrotactic microorganisms and thermoelectric effects on the dynamics of $29 \mathrm{~nm} \mathrm{CuO-water} \mathrm{nanofluid} \mathrm{over} \mathrm{an} \mathrm{upper} \mathrm{horizontal} \mathrm{surface} \mathrm{of} \mathrm{paraboloid}$ of revolution. Multidiscipline Modeling in Materials and Structures, 14(4), 695-721.

[20] Makinde, O. D., \& Animasaun, I. L. (2016). Bioconvection in MHD nanofluid flow with nonlinear thermal radiation and quartic autocatalysis chemical reaction past an upper surface of a paraboloid of revolution. International Journal of Thermal Sciences, 109, 159-171.

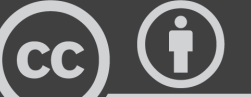

BY

(C) 2019 by the authors; licensee PSRP, Lahore, Pakistan. This article is an open access article distributed under the terms and conditions of the Creative Commons Attribution (CC-BY) license (http://creativecommons.org/licenses/by/4.0/). 